\title{
COVID19: Inflammatory biomarkers, predictors of severity?
}

\author{
R. Aniq Filali *, M. Kassimi**, R. Jabbouri, A. Naïtlho *** \\ DOI: 10.29322/IJSRP.10.08.2020.p10444 \\ http://dx.doi.org/10.29322/IJSRP.10.08.2020.p10444
}

\begin{abstract}
In December 2019, a viral pneumonia caused by the severe acute respiratory syndrome coronavirus 2 (SARS-COV-2), begins in Wuhan (China) and quickly spread around the world. Today more than 3.5 million people are infected. Even though the mortality rate is relatively low, a considerable number of patients die every day. It appears that the cytokine storm is one of the main causes of death in coronavirus disease 2019 (COVID-19), and that targeting the inflammation process would save the most severe patients. Our experience at the hospital has shown us that it is still difficult to recover patients at an advanced stage of the disease. Since biology is more sensitive than imaging in predicting disease severity, monitoring of inflammatory biomarkers such as serum ferritin, D-dimer and lactate dehydrogenase (LDH), has great value in the early detection of patients at risk of developing acute respiratory distress syndrome (ARDS) or multisystemic failure. These findings may be useful with the imminent risk of a second outbreak for a correct management of patients with COVID19.
\end{abstract}

\section{Introduction}

Coronaviruses are enveloped, single positive stranded RNA viruses, which belong to the subfamily Coronavirinae. While the majority of these viruses are responsible for mild infections, there have been two past epidemics caused by betacoronaviruses. The Severe Acute Respiratory Syndrome Coronavirus (SARS-CoV) and the Middle East Respiratory Syndrome Coronavirus (MERS-CoV), resulted in more than 10,000 cases in the past two decades, with a mortality rate of $10 \%$ for SARS-CoV and $37 \%$ for MERS-CoV.

At the end of December 2019, numerous cases of viral pneumonia were recorded in Wuhan, a city in the Hubei province of China, and were associated with a seafood market that also sold non-aquatic animals. On 7 January, a new coronavirus was identified, and named COVID-2019 by the WHO.

Morocco, like the rest of the world, is facing this new infection caused by the coronavirus. The country experienced its first case in early March 2020, and quickly many sanitary measures were put in place by the authorities, such as containment and early detection of suspicious cases. It is in this context that the structure and staff of the Cheikh Khalifa University Hospital (HCK) were reorganized to cope with a large influx of patients. 
The Internal Medicine team has been involved in the management of this health crisis, as it is a multifaceted pathology. While its tropism is primarily pulmonary, in some patients, other organs may also be affected, as a consequence of a systemic inflammatory reaction. It appears that the cytokine storm is one of the main causes of death in coronavirus disease 2019 (COVID-19), and that targeting the inflammation process would save the most severe patients.

Here we present an analysis of the temporal changes of inflammatory biomarkers during hospitalization in patients with COVID19, to highlight their interest in predicting the severity of the disease and in guiding therapeutic management.

\section{Materials and methods}

\section{Study design and participants}

This retrospective study was carried out in the internal department of the Cheikh Khalifa University Hospital in Casablanca from March 23th, 2020 to April 27th.

All patients diagnosed as having COVID-19according to WHO interim guidance, hospitalized at the HCK, dead or discharged; were enrolled in this study.

Patients hospitalized in COVID units with a positive chest computed tomography (CT) scan and a biological inflammatory syndrome but a negative real-time reverse-transcriptase-polymerase chain reaction (rRT-PCR), have been excluded.

\section{Data collection}

Patients admitted on suspicion of COVID-19 were routinely provided with an rRT-PCR treated by nasopharyngeal swab, a CT scan, and a revised and completed blood examination as per the new data in the literature. Some patients were transferred from other hospitals and benefited from some of these examinations on an outpatient basis.

Routine blood examinations included blood count, C Reactive Protein (CRP), LDH, serum ferritin, D-dimer, kidney and liver function, and myocardial enzymes.

Epidemiological, demographic, clinical, laboratory, treatment, and outcome data were extracted from electronic medical records using a standardized data collection form.

Missing data were retrieved from the physical records recovered from archives.

\section{Statistical analysis}

We used the IBM SPSS statistics software for the statistical analysis of our data and to explore the biological risk factors associated with death during hospitalization. 
Qualitative variables were expressed in headcount and percentage, and quantitative variables were expressed in mean and standard deviation.

The student T-test was used to compare two means. The significance level was set at $\mathrm{p}<0.05$.

\section{Results}

Out of 631 patients tested before April 27, 2020, 146 were confirmed positive and were hospitalized in HCK's COVID units. The median age was 49.16 years, ranging from 5 to 84 years, and males were more frequently affected (59\%). Comorbidities existed in 91 of the patients, hypertension being the most common, followed by diabetes. $71.9 \%$ of patients had non-severe forms (mild and moderate) versus $28.1 \%$ severe (severe and critical). The most common symptoms on admission were cough and fever. The majority of patients received first-line treatment (Plaquenil-Azythromicine combination), unless contraindicated (4 cases). Antivirals (Lopinavir / Ritonavir) were administered to 11 patients, including 6 in the intensive care unit. Tocilizumab (a blocker of IL-6R) was also used in the intensive care unit in 5 patients.

We recorded 14 deaths. These deaths were the consequence of complications such as acute respiratory distress syndrome (ARDS), septic shock, heart failure or multisystemic failure (Figure 1).

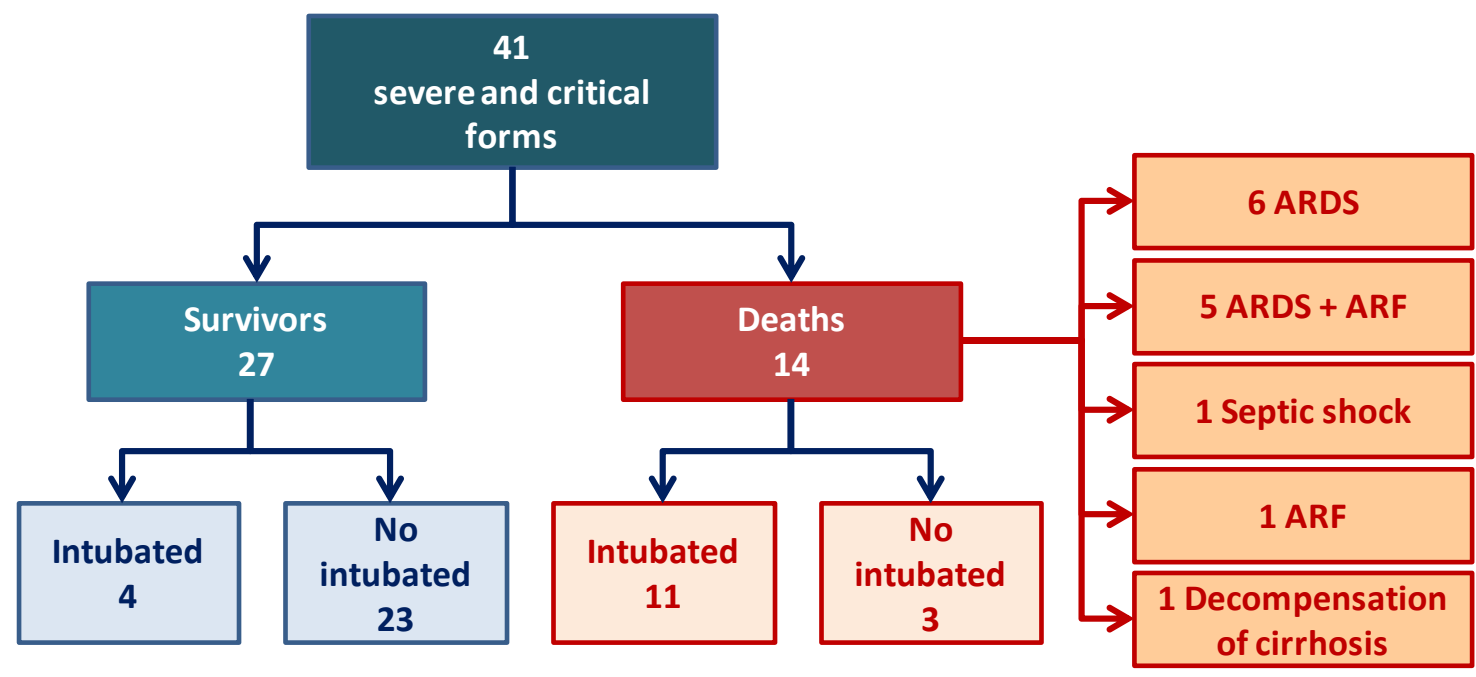

\section{Figure 1: Status of patients in intensive care units, and main complications leading to the 14 deaths}

ARDS: acute respiratory distress syndrome

ARF: acute renal failure

It is important to note that patients admitted to the intensive care unit had elevated white blood cell and neutrophil counts, lymphopenia, and increases in CRP, D-dimer, ferritin and LDH. If we look at the group of deceased patients, we see that all these 
parameters are disturbed and are accompanied at the last check-ups by the installation of cytolysis, acute renal failure and an increase in troponins, the signature of multisystem failure (Figure 2).

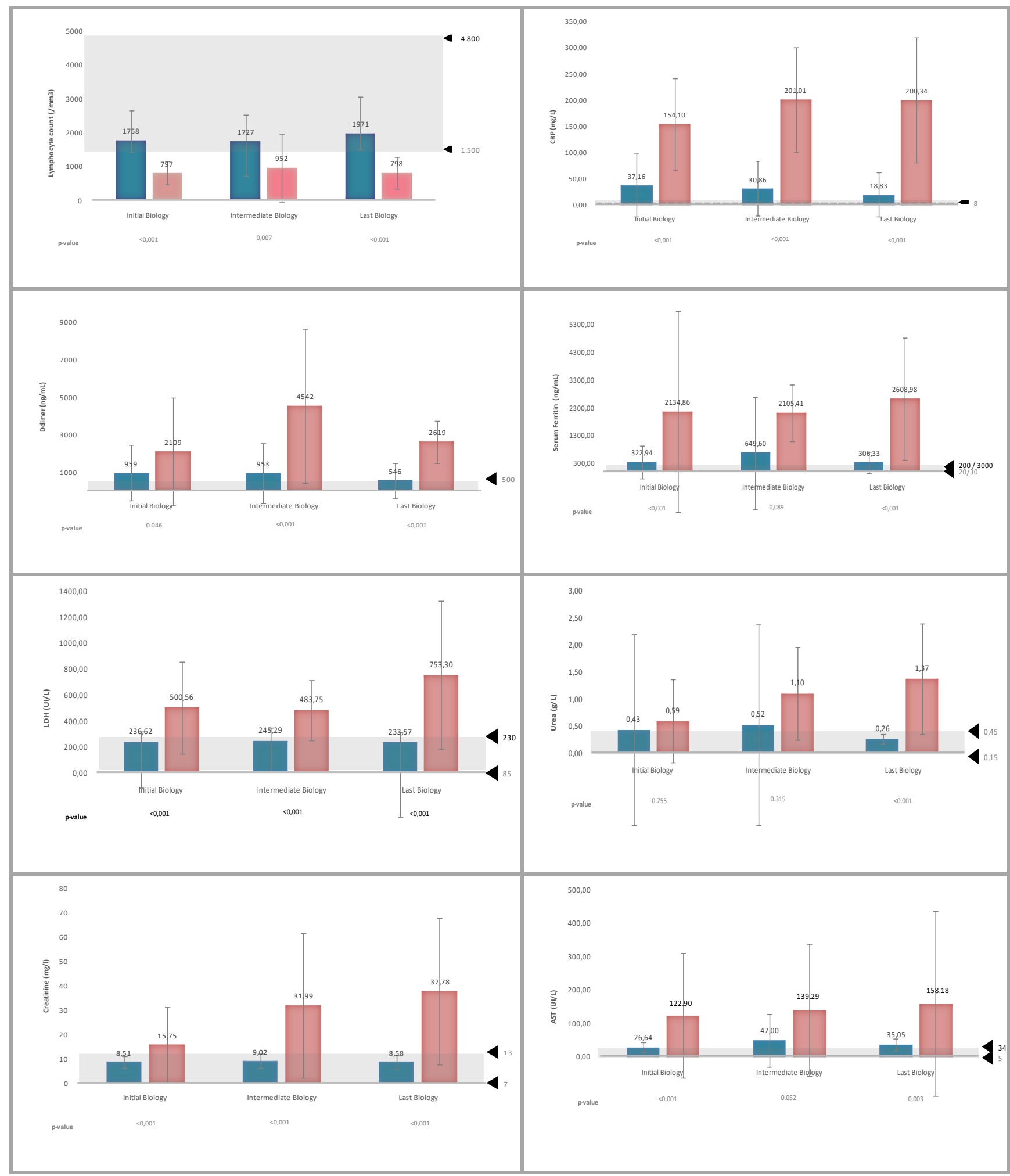




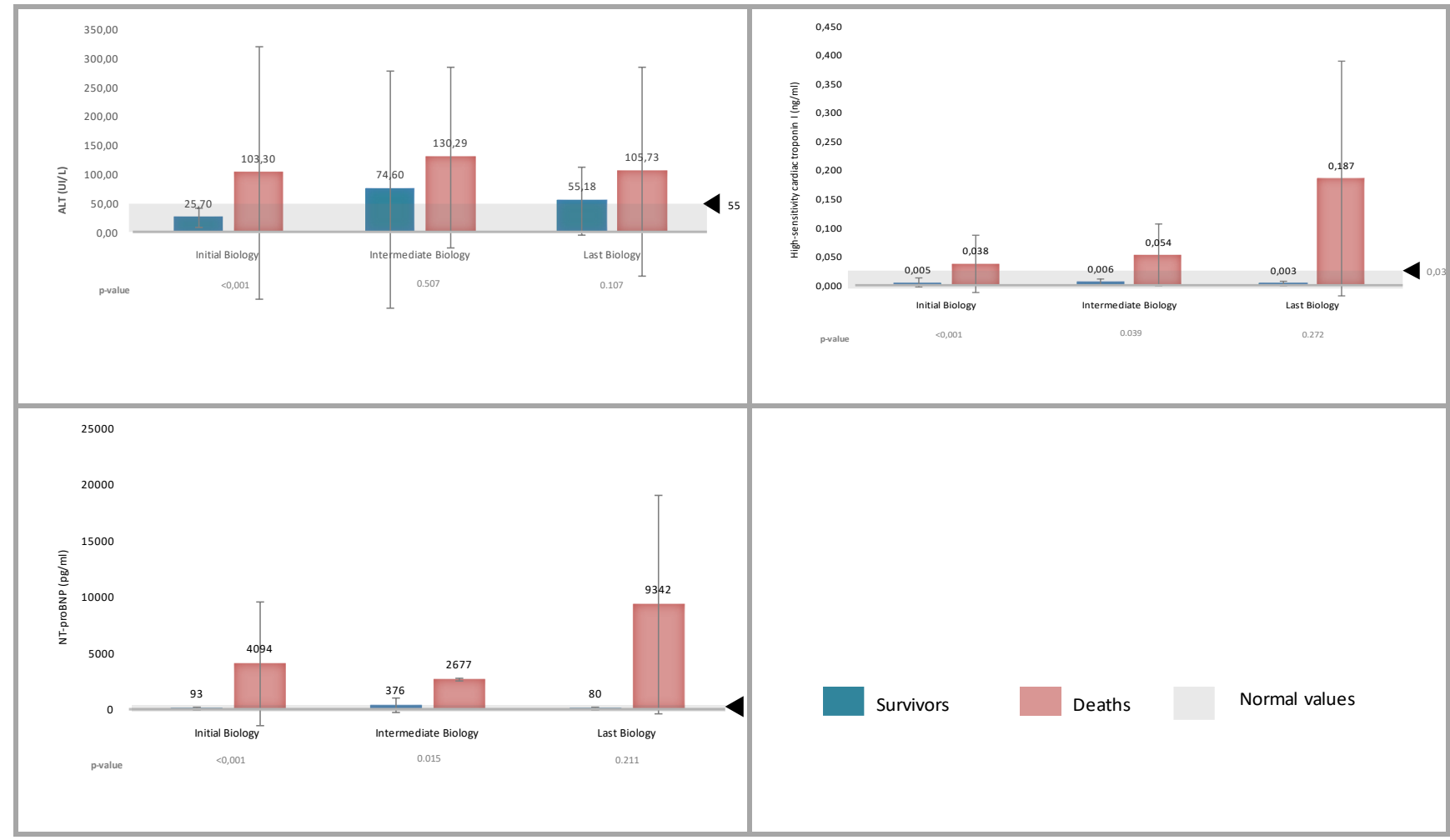

\section{Figure 2: Temporal changes in laboratory markers in COVID-19 patients}

The markers were tracked from the first day of the patients' hospitalization. We evaluated their temporal changes, based on three blood examinations: initial, intermediate and last one. Differences between survivors and non-survivors were significant from illness onset.

These biological perturbations highlight the existence of systemic inflammation downstream of the infection, and reflect the transformation of the infectious disease into a potentially fatal systemic inflammatory disease.

\section{Discussion}

Our results seem to agree with the literature. Zhou et al [1], in a retrospective cohort study identified several risk factors for death in patients with COVID-19 who were hospitalized in Wuhan, like older age, comorbidities, SOFA score, higher rates of D-dimer $(1 \mu \mathrm{g} / \mathrm{ml})$, but also elevated levels of ferritin, LDH, IL6, cardiac troponins, and lymphopenia. The elevation of the inflammatory biomarkers would indicate the transition from infectious to systemic disease.

There are two overlapping pathological phenomena, the first is triggered by the virus, and the second is an inflammatory response of the host. In all individuals regardless of age or comorbidities, the disease tends to present these two phases, although at different levels of severity.

The better understanding of the pathophysiology of SARS-COV2 $[2 ; 3 ; 4]$, has allowed us to consider the use of immunosuppressants in COVID-19 in addition to anti-viral therapy [5; 6]. Indeed, this infection reminds us of certain vasculitis 
whose etiopathogeny involves certain viruses such as $\mathrm{HCV}$ or HBV, and where it is important to target inflammatory events downstream of the infection.

The problem we faced was to know at witch stage of the disease this therapeutic approach should be used. If high-dose corticosteroid therapy has been administered systematically in patients with a persistent elevation of ferritinemia, LDH or DDimer, Tocilizumab (anti-IL6) could only be used in 5 patients in intensive care at the intubation-ventilation stage and the results were disappointing as we recorded 4 deaths.

It is therefore essential from a practical point of view to establish a clearer course of action, based on the monitoring of severity factors and essentially the biological ones which reflect the onset of the systemic inflammatory syndrome responsible in certain patients for respiratory distress or even multi-organ failure. The superiority of biology over imaging in the prediction of severity has been noted. If CT seems to be more sensitive than viral DNA search by RT-PCR for COVID-19 diagnosis [7; 8]; there is no clinical-radiological correlation. In fact, subjects with few symptoms may have CT abnormalities similar to those of patients with more severe forms of the disease (Figure 3).

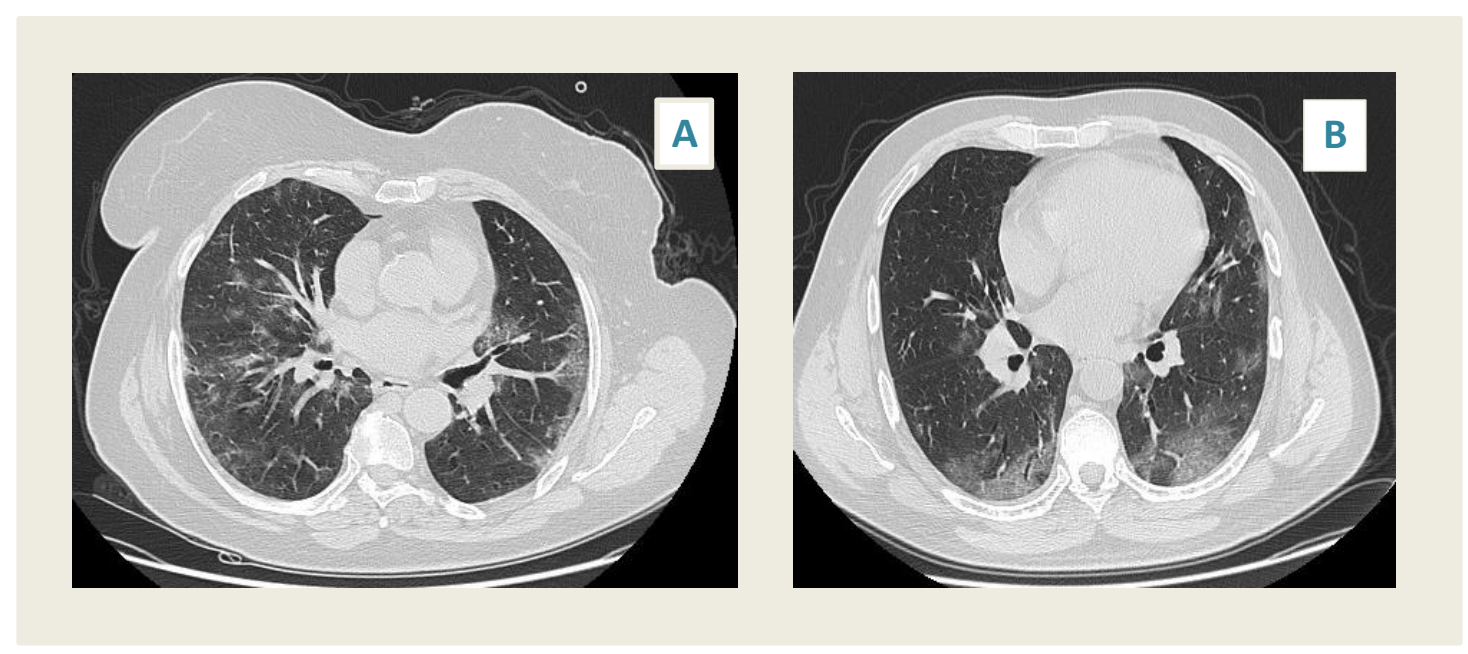

Figure 3: CT scan with CORADS 5 in patients with mild form of COVID-19

Axial CT images without intravenous contrast corresponding to a CORADS 5, of a 57 years old woman (A) and a 55 years old man (B) who presented a mild form of the disease.

The scan showed bilateral peripheral areas of GGO (Ground glass opacities).

It is very high level of suspicion for pulmonary involvement by COVID-19.

The clinical-therapeutic classification proposed by Siddiqi et Mehra [9], seems to be the most appropriate in practice. This classification distinguishes three degrees of severity in patients with COVID19, corresponding to distinct clinical and biological conditions, each involving specific therapeutic management.

The first stage occurs at the time of inoculation of the virus responsible for the onset of the disease. SARS-COV-2 binds to the angiotensin converting enzyme 2 (ACE2) receptor located predominantly in the alveoli (pneumocytes 2) and multiplies in the 
lungs during the incubation period [10]. Patients, who limit the infection to this stage, have mild clinical symptoms and make a remarkable recovery. Antiviral treatment at this stage allows accelerated control of viremia.

This is followed by the stage where lung disease is established as a result of the multiplication of the virus and the localized inflammation it causes. Clinically, this translates into a viral pneumonia with cough, fever and in some cases dyspnea. It is generally at this stage that the radiological abnormalities can be assessed, particularly in the thoracic CT scan. Markers of inflammation may be increased, but usually only moderately. If patients develop hypoxia or a significant increase in the inflammatory syndrome, anti-inflammatory therapy may be used to slow the progression of patients to mechanical ventilation.

Only a minority of patients experience the 3rd stage, which is characterized by the development of cytokine release syndrome (CRS), which is a hyper-inflammatory syndrome characterized by fulminant and potentially fatal hypercytokineemia [11]. If the dosage of interleukins like IL6 who plays an important role in CRS could not be performed, we observed an accentuation of lymphopenia, which reflects a massive recruitment of $\mathrm{T}$ lymphocytes; or a significant increase in inflammatory biomarkers such as CRP, LDH, Ferritin and Ddimers. In addition to alveolar cells, ACE2, the receptor for SARS-COV-2 is expressed on vascular endothelial cells which would explain the involvement of other organs, as in systemic vasculitis. Anti-inflammatory therapy is then required to reduce systemic inflammation before it leads to multisystemic failure. High-dose corticosteroid therapy and Tocilizumab (anti-IL6) are then recommended [12]. The increase in D-dimers reflects an alteration in blood coagulability. Indeed, patients end up presenting numerous microthrombi disseminated in the organism probably responsible for the multivisceral failure. As for the ARDS, it is probably the consequence of a pulmonary intravascular coagulopathy (PIC). It would seem that in the context of this systemic hyperinflammation, patients would develop antiphopholipid Antibodies [13]. If an anticoagulant propilaxy is systematically administered to all patients as soon as they are hospitalized, it is essential to associate in this minority of patients a Low-molecular-weight heparin (LMWH) at a curative dose.

Our experience has shown us that despite the use of this therapeutic arsenal, the prognosis remains very engaged and patient recovery is complicated. However, it suggests that the deployment of these therapies should be earlier, hence the interest in monitoring inflammatory biomarkers to help us detect candidate patients in time [14], avoid their hospitalization in intensive care units where the mortality rate is high [15].

The major limitation of this study was that not all laboratory tests were done in all patients, given that the guidelines were not initially clearly defined.

\section{Conclusion}

Inflammatory biomarkers could therefore be reliable predictive factors for the detection of potentially serious patients, in order to adapt their therapeutic management and improve their prognosis. 
Targeted drug therapy against the virus is most promising when applied early in the course of the disease, but its usefulness at an advanced stage is questionable. The use of anti-inflammatory therapy too early may not be necessary and may even promote viral replication, but it is essential in the systemic inflammatory stage to slow the onset of ARDS and multisystemic failure.

\section{Declarations}

This research did not receive any funding.

This study was approved by the Ethics committee of Cheikh Khalifa Hospital.

We declare no conflict of interest.

\section{References}

[1] F. Zhou, T. Yu, R. Du, G. Fan, Y. Liu, Z. Liu, et al. Clinical course and risk factors for mortality of adult inpatients with COVID-19 in Wuhan, China: a retrospective cohort study. Lancet 2020; 395: 1054-62, https://doi.org/10.1016/

[2] E. De Wit, N. van Doremalen, D. Falzarano, V. J Munster. SARS and MERS: recent insights into emerging coronaviruses. Nat Rev Microbiol (2016), doi: 10.1038/nrmicro.2016.81

[3] L. Mousavizadeh and Sorayya Ghasemi. Genotype and phenotype of COVID-19: Their roles in pathogenesis. Journal of Microbiology, Immunology and Infection, https://doi.org/10.1016/j.jmii.2020.03.022

[4] Rothan and Byrareddy, 2020. H.A. Rothan and S.N. Byrareddy. The epidemiology and pathogenesis of coronavirus disease (COVID-19) outbreak. Journal of Autoimmunity (2020), https://doi.org/10.1016/j.jaut.2020.102433

[5] W. Zhang, Y. Zhao, F. Zhang, Q. Wang, T. Li, Z. Liu, et al. The use of anti-inflammatory drugs in the treatment of people with severe coronavirus disease 2019 (COVID-19): The experience of clinical immunologists from China. Clinical Immunology (2019), https://doi.org/10.1016/j.clim.2020.108393

[6] D. Marotto, P. Sarzi-Puttini. What is the role of rheumatologists in the era of COVID-19? Autoimmunity Reviews (2020), https://doi.org/10.1016/j.autrev.2020.102539

[7] X. Xie, Z. Zhong, W. Zhao, C. Zheng, F. Wang F, J. Liu . Chest CT for Typical 2019-nCoV Pneumonia: Relationship to Negative RT-PCR Testing. Radiology (2020)

[8] T. Ai, Z. Yang, H. Hou, et al. Correlation of Chest CT and RT-PCR Testing in Coronavirus Disease 2019 (COVID19) in China: A Report of 1014 Cases. Radiology (2020)

[9] HK Siddiqi, MR Mehra. COVID-19 Illness in Native and Immunosuppressed States: A Clinical-Therapeutic Staging Proposal. Journal of Heart and Lung transplantation (2020). Doi:10.1016/j.healun.2020.03.012 
[10] F. Rivellese, E. Prediletto. ACE2 at the centre of COVID-19 from paucisymptomaticinfections to severe pneumonia. Autoimmunity Reviews (2020), Article 102536

[11] C. Zhang, Z. Wu, JW. Li, H. Zhao, GQ. Wang. The cytokine release syndrome (CRS) of severe COVID-19 and Interleukin-6 receptor (IL-6R) antagonist Tocilizumab may be the key to reduce the mortality. International Journal of Antimicrobial Agents (2020), Article 105954

[12] A Prospective, Randomized, Factorial Design,Interventional Study to Compare the Safety and Efficacy of Combinations of Blockade of Interleukin-6 Pathway andInterleukin-1 Pathway to Best Standard of Care inImproving Oxygenation and Short- and Long-termOutcome of COVID-19 Patients With Acute HypoxicRespiratory Failure and Systemic Cytokine Release. ClinicalTrials.gov identifier: NCT04330638

[13] Y. Zhang, M. Xiao, S. Zhang, Y. Li. Coagulopathy and Antiphospholipid Antibodies in Patients with Covid-19. New Engl J Med (2020). DOI: 10.1056/NEJMc2007575

[14] Early Institution of Tocilizumab Titration in Non-CriticalHospitalized COVID-19 Pneumonitis. ClinicalTrials.gov identifier: NCT04331795

[15] PK. Bhatraju, BJ. Ghassemieh, M. Nichols, R. Kim, K R. Jerome, A K. Nalla, et al. Covid-19 in Critically Ill Patients in the Seattle Region-Case Series. New Engl J Med (2020). DOI: 10.1056/NEJMoa2004500

\section{Authors}

First Author - R. ANIQ FILALI, Dr, Department of Internal Medicine, Cheick Khalifa International University Hospital, Mohammed VI University of Health Sciences (UM6SS), Casablanca, Morocco. rita.filali.7@gmail.com

Second Author - M. KASSIMI, Dr, Department of Radiology, Cheick Khalifa International University Hospital, Mohammed VI University of Health Sciences (UM6SS), Casablanca, Morocco. meriem.kassimi@live.fr

Third Author - R. JABBOURI, A. NAÏTLHO, Pr, Department of Internal Medicine, Cheick Khalifa International University Hospital, Mohammed VI University of Health Sciences (UM6SS), Casablanca, Morocco. rajaa.jabbouri@gmail.com, mnaitlhou@gmail.com

Correspondence Author - R. ANIQ FILALI, rita.filali.7@gmail.com, 00212661497827. 